EPJ Web of Conferences 52, 11003 (2013)

DOI: 10.1051/epjconf/20135211003

(C) Owned by the authors, published by EDP Sciences, 2013

\title{
New detection technologies for ultra-high energy cosmic rays and neutrinos
}

\author{
Sebastian Böser ${ }^{1, a}$ \\ ${ }^{1}$ Univ. Bonn, Germany
}

\begin{abstract}
Even with an accumulated data set from an integrated six years of lifetime from the Auger experiment, no point sources of charged cosmic rays have be identified at the highest energies. Significantly increased apertures such as promised by the JEMEUSO mission will be required to identify these sources from the cosmic ray signatures themselves. However, in employing water-cherenkov surface detectors as well as fluorescence telescopes, Auger has demonstrated the power provided by the hybrid technology approach. New detection technologies thus provide a valuable tool, in particular for the study of systematic effects.

Over the past decade, in particular radio detection of cosmic ray air-showers has become a viable future detection technology to enhance and complement existing air-shower experiments. Following the proof-of-principle provided by the Lopes experiment, this technology is now being pursued in all major air-shower detectors. In the $\mathrm{MHz}$ regime, the radio signal is dominated by geomagnetic emission from the electrons deflected in the earth magnetic field, with secondary contributions from a global charge excess. As the majority of the energy in the shower is carried by these electron and the radio signal traverses the atmosphere basically unattenuated, this approach not only promises superior energy resolution but may also provide an independent handle on the longitudinal shower development and hence the primary composition. Theoretical signal predictions provided by detailed Monte-Carlo simulations as well as analytic shower parametrizations are in good agreement with measurements provided by the AERA and Codalema experiments. Recent efforts also include studies of the radio emission in the $\mathrm{GHz}$ regime, where the ambient noise is significantly reduced, yet the emission mechanism in this regime has not been firmly established yet.

As neutrinos are not deflected in the intergalactic magnetic fields, the detection of neutrino-induced cascades in dense media provides another promising approach for the identification of the sources of cosmic rays. The low event rates and large required target volumes limit the experimental methods to far-ranging signatures from the cascade, such as acoustic emission from the quasi-instantaneous energy deposit or Cherenkov emission from the charged particles in the cascade. Searching for optical Cherenkov photons in a cubic-kilometer of Antarctic ice, the IceCube experiment has recently found an excess of high-energy neutrinos in the TeV-PeV range. Yet its effective volume is too small to detect the GZK flux predicted from interaction of the highest-energy cosmic rays with the ambient cosmic microwave background. Seeking to increase the observed target volume, radio observations of the rim of the moon have energy thresholds well beyond the EeV scale and thus are more likely to find interactions of charged cosmic rays than GZK
\end{abstract}

asboeser@physik.uni-bonn.de

This is an Open Access article distributed under the terms of the Creative Commons Attribution License 2.0, which permits unrestricted use, distribution, and reproduction in any medium, provided the original work is properly cited. 
neutrinos. The currently best sensitivity to this flux is provided from searches for $\mathrm{GHz}$ radio emission of neutrino-induced cascades in the antarctic ice from the ANITA ballon experiment. While no high-energy neutrinos have been found, a geomagnetic emission component from air-showers

\section{Slides}

The slides of the talk can be found on the website of the symposium ISVHECRI 2012: https://indico.desy.de/conferenceOtherViews.py?view=standard\&confId $=4594$ 